\title{
LA ARTICULACIÓN DE LA HEGEMONÍA NEOLIBERAL A TRAVÉS DE LAS POLÍTICAS EDUCATIVAS
}

\author{
Jezabel Rodríguez Pérez \\ Universidad Autónoma de Barcelona \\ Jezabel_Rodguez@hotmail.com
}

\section{Resumen}

La presente investigación tiene como principal propósito analizar la articulación de la hegemonía neoliberal a través de las políticas educativas. Considerando el discurso como eje articulador de hegemonías, se ha procedido al examen de sus condiciones sociales de producción, de los elementos semiótico-ideológicos imperantes y de las áreas de intervención. El Análisis Discursivo nos ha permitido constatar que el actual sistema educativo se fundamenta sobre la racionalidad instrumental del neoliberalismo y las biopolíticas de control cuantitativo del rendimiento. Si la eficacia se constituye como criterio de evaluación interna del espacio académico, el principio de adaptación al mercado se constituye como mecanismo de regulación externa.

Palabras clave: hegemonía, biopolítica, educación, Análisis Discursivo, neoliberalismo.

\section{THE ARTICULATION OF NEOLIBERAL HEGEMONY THROUGH EDUCATIONAL POLICIES}

Abstract

The main purpose of this research is to analyze the articulation of neoliberal hegemony through educational policy. Considering discourse as the articulating axis of hegemonies, we have proceeded to examine its social conditions of production, the prevailing semiotic-ideological elements and the areas of intervention. The Discursive Analysis has allowed us to verify that the current educational system is based on the instrumental rationality of neoliberalism and the biopolitics of quantitative control of performance. If the effectiveness is constituted as criterion of internal evaluation of the academic space, the principle of adaptation to the market is constituted as a mechanism of external regulation.

Keywords: hegemony, biopolitics, education, Discursive Analysis, neoliberalism.

DOI: http://doi.org/10.25145/j.laguna.2018.42.006

ReVista Laguna, 42; julio 2018, pp. 95-117; ISSN: e-2530-8351 


\section{INTRODUCCIÓN}

La educación ciudadana ha tenido un rol en la reflexión filosófica tan destacado que entronca con los orígenes del mismo filosofar. Considerando que la buena organización político-cívica requiere una formación adecuada de los individuos que habitan en ella, la tradición sofística y el mismo Platón, entre otros, desarrollaron modos de concebir la función educativa, el saber y el conocimiento, distintivamente ${ }^{1}$. Dada la actual problemática educacional de la sociedad española, el presente trabajo tiene como principal propósito analizar la articulación de las hegemonías sociales a través de las políticas educativas; más concretamente, se estudiará cómo la hegemonía neoliberal -por ser considerada la forma hegemónica dominante en la actualidad-repercute en el ámbito educativo. Ello supone tomar en consideración dos puntos de partida centrales: por un lado, la concepción de la escuela $-\mathrm{y}$, por ende, del sistema educativo- como aparato ideológico del Estado; por otro lado, las definiciones de hegemonía y neoliberalismo otorgadas por A. Gramsci y M. Foucault respectivamente.

Teniendo esto en cuenta, el texto que aquí presentamos dispondrá de dos apartados principales: un primer apartado que engloba, por un lado, los postulados de la teoría marxista concernientes a la relación Estado-escuela y a los procesos hegemónicos; un segundo apartado destinado al análisis de la articulación hegemónica neoliberal y su incidencia en la racionalidad gubernamental y en las políticas educativas de los últimos años. Para ello, se responderá a dos cuestiones básicas: primero, cuáles son las características que definen la racionalidad gubernamental o el quehacer estatal; y segundo, de qué manera se estudia la articulación de los procesos hegemónicos, incluyendo la construcción de las subjetividades sociopolíticas.

Con respecto al primer punto, se recurrirá al método genealógico de Foucault y sus estudios sobre las variables contextuales histórico-económicas que han contribuido a definir la racionalidad gubernamental neoliberal. En relación con el segundo punto, se utilizará el Análisis del Discurso, la teoría de E. Laclau y su reactualización de la concepción gramsciana de hegemonía. Dado que, de acuerdo con Laclau y Mouffe, las condiciones de posibilidad hegemónica se articulan en un espacio simbólico-discursivo, el Análisis del Discurso será tomado como instrumento metodológico clave. En este caso, dos son los elementos discursivos que se tendrán en cuenta: 1) elementos extralingüísticos, relativos a la intencionalidad de los emisores y a las tecnologías de gobierno que estructuran el poder hegemónico; 2) elementos lingüísticos, concernientes a las redes conceptuales utilizadas explícitamente para crear lógicas de equivalencia y patrones de valoración dentro y fuera del escenario formal educativo.

Puesto que el análisis discursivo se desarrolla en el plano funcional de los enunciados neoliberales, políticos y educativos, por un lado, y en la dimensión estructural de la organización académico-escolar, por otro, la metodología procederá

1 Barrio, J.: Historia de la filosofía, Barcelona, Vicens-vives, 1989, pp. 1-43. 
a su estudio en dos niveles interdependientes: el nivel del sujeto, esto es, la fuente emisora y reproductora de la lógica neoliberal; y el nivel del objeto, es decir, las políticas e instrumentos que diseñan y valoran el proceso de enseñanza/aprendizaje. De este modo, el Análisis Discursivo posibilitará examinar cómo la política educativa neoliberal articula su discurso hegemónico reconfigurando la concepción del sujeto de aprendizaje, así como la finalidad de los objetivos educativos, integrando o excluyendo, según convenga, determinados perfiles psicopedagógicos. Los mecanismos de evaluación serían considerados, con todo ello, como tecnologías de control encargados de asegurar la fortaleza de la estructura semiótica o discursiva neoliberal.

Finalmente, sobre la base de los análisis elaborados se establecerán una serie de disquisiciones críticas que permitirán discernir de qué forma el Estado y la sociedad civil se ven determinados por la lógica neoliberal ${ }^{2}$, observando hasta qué punto las políticas educativas (sus finalidades y modos de evaluación) y las subjetividades que generan (homo economicus) responden a dicha lógica. A este respecto, el estudio de la estructura político-económica como condición determinante de la configuración del espacio estatal y, por tanto, del diseño de las políticas educativas tendrá en consideración la siguiente relación de influencias: neoliberalismo-Estadopolítica educativa, donde la política educativa, en la medida en que adquiere una morfología neoliberal, se erige como legitimadora de su hegemonía.

La pérdida de legitimidad soberana y las posibles respuestas contrahegemónicas que desde la esfera educativa puedan fraguarse son dos consideraciones finales que, a modo de conclusión, trazarán algunas vías abiertas a reflexionar en nuestro presente y futuro.

\section{LAS BASES LIBERALES Y NEOLIBERALES DEL ESTADO MODERNO}

Dado que el marco legislativo de las sociedades actuales se fragua en la institución estatal, estudiar la articulación hegemónica neoliberal a través de las políticas educativas requiere, primeramente, un acercamiento a la conformación de la racionalidad gubernamental de las últimas décadas. Para ello nos valdremos de los análisis de Michel Foucault expresados mayormente en la obra Nacimiento de la biopolítica (1979), pues estos desbrozan la incidencia del neoliberalismo sobre el quehacer del gobierno. En virtud de estos aspectos, los próximos apartados se distribuirán en dos bloques. Por un lado, se indagarán las causas por las que la lógica económica-neoliberal fue integrada en ciertas esferas sociales estimadas tradicionalmente «no económicas», tales como el ámbito educativo. Teniendo en cuenta que la

${ }^{2}$ La expresión «lógica neoliberal», inspirada en las reflexiones de Foucault en el Nacimiento de la biopolítica, se utilizará en el presente trabajo para aludir a una forma de pensamiento o «sentido común» propio del neoliberalismo. Foucault, M.: Nacimiento de la biopolítica: curso en el Collége de France (1978-1979). Buenos Aires, Fondo de Cultura Económica, 2007. 
configuración formal del sistema educativo se ejerce desde el espacio gubernamental, se analizará por qué este ha modificado su posición de responsabilidad política con respecto a las diversas áreas sociales correlativamente al fortalecimiento de posturas económico-neoliberales. Por otro lado, en un segundo apartado estudiaremos qué estrategias, mecanismos o modelos enunciativos fueron utilizados -y se utilizan-para permitir la expansión de la lógica económico-liberal en la escuela. En este sentido, se discernirán los procesos hegemónicos discursivos que han posibilitado la articulación de dispositivos ideológicos neoliberales en el sistema educativo.

\subsection{El Estado MOderno y la Racionalidad GUbERnAMENTAL}

El «corpus teórico» del Nacimiento de la biopolitica otorga, grosso modo, dos claves principales a tener en consideración en nuestros análisis, a saber: primero, los fundamentos que engendraron la aparición del Estado moderno y contemporáneo, esto es, las vicisitudes de la legislación jurídica iusnaturalista y la política económica liberal; segundo, la reconfiguración del espacio de acción gubernamental según las demandas liberales y neoliberales de las últimas décadas. De ahí que Foucault, antes de embarcarse en la disquisición conceptual de la noción «biopolítica», subraye la importancia de abordar la coyuntura histórico-social entretejida por dos pilares elementales del Estado moderno: la economía política y la jurisprudencia ${ }^{3}$.

En el estudio de las diversas formas de gobierno acaecidas a partir de los siglos XVI y XVII, Foucault distingue dos dimensiones de gobernabilidad primordiales: la política interna, relativa a medidas organizadoras y legitimadas dentro del Estado-nación; y la política externa, referida a las relaciones interestatales ${ }^{4}$. Este doble espacio de gobernabilidad, acorde con Foucault, permite detectar el principal elemento diferenciador entre la modalidad gubernamental del Medioevo y el Estado moderno: los límites de legitimidad de la acción soberana ${ }^{5}$. Y es que, allá donde la soberanía medieval promovía la expansión ilimitada de su gobierno, es decir, pretendía detentar interna y externamente el dominio político, el Estado moderno integró una racionalidad gubernamental basada en el respeto de las fronteras interestatales. Consecuentemente, la política externa adquiere un cariz más restrictivo en pos del convenio pacífico con otras naciones ${ }^{6}$.

Ahora bien, ¿cuál fue el elemento causal que predispuso a tal variación de las pretensiones gubernamentales? Para Foucault, el factor parece evidente: el mercantilismo ${ }^{7}$. Si bien a finales del siglo XVII y principios del siglo XVIII aparece la necesidad de limitar la prâxis estatal sobre la base del derecho, a partir del siglo XVIII nace una nueva forma de poner límites al poder interno del gobierno, esto es, la

${ }^{3}$ Foucault, M.: op. cit., pp. 21-30.

${ }^{4}$ Ibidem, pp. 20-23.

5 Ibidem, p. 21.

${ }^{6}$ Ibidem.

7 Ibidem, p. 30. 
economía política ${ }^{8}$. A este respecto, cabe añadir que las nuevas concepciones de racionalidad económico-monetaria vienen de la mano de dos instrumentos básicos de control social: la policía, encargada de garantizar la gobernabilidad interna; y el ejército, destinado a la defensa interestatal ${ }^{9}$. Así pues, consolidado cierto marco de seguridad político-civil dentro y fuera de las fronteras del Estado-nación, las actividades mercantiles cobran una mayor fuerza operativa, demandando instancias de regulación o desregulación según el caso.

En razón de los límites que adquieren los espacios de actuación gubernamental, Foucault señala lo siguiente: mientras que las instituciones judiciales se vuelven agentes externos en la delimitación del Estado, concibiéndose anteriores y constitutivas del mismo ${ }^{10}$, la economía política aparece como criterio delimitador de la lógica interna del gobierno o, en palabras de Foucault, como «regulación interna de la racionalidad gubernamental $\aleph^{11}$. De esta manera, a partir del s. XVIII surge un nuevo mecanismo de racionalidad de gobierno que reformula el campo de responsabilidad política en términos prescriptivos; las áreas de intervención y control estatal quedan, así, sujetas a un «deber ser», esto es, una lógica de actuación evaluada en la práctica sobre la base de una serie de objetivos o principios que determinan el Estado contemporáneo ${ }^{12}$.

A tenor de esta última consideración, cabe apuntar que la reorganización de la práctica gubernamental no se da fundamentalmente en los sujetos sino en «las cosas por hacer»; de ahí que los límites de la acción no estén trazados en los sujetos propiamente dichos (legisladores), sino en la prâxis política (legislaciones) ${ }^{13}$. He ahí la razón por la cual la nueva racionalidad gubernamental, más allá de criticar el abuso de un soberano particular, se enfoca en el control de los procedimientos legislativos. En este sentido, Foucault estima que «toda esta cuestión de la razón gubernamental crítica va a girar alrededor del cómo no gobernar demasiado ${ }^{14}$, presupuesto de sello eminentemente liberal. El liberalismo político-económico aparece, así, como límite constituyente del Estado contemporáneo. Este surgimiento del liberalismo políticoeconómico como criterio de una nueva racionalidad gubernamental requirió, acorde con el filósofo francés, un "régimen de verdad ${ }^{15}$, es decir, unos dispositivos ideológicos que predispusieran la aparición, integración y consolidación de la lógica liberal como fundamento del «buen» gobierno. Es aquí donde, a nuestro entender, radicó la arena de la lucha hegemónica, el espacio político-civil en el que se construyeron

8 Ibidem.

${ }^{9}$ Ibidem, p. 21.

${ }^{10}$ Ibidem, p. 24.

${ }_{11}$ Ibidem, p. 26.

${ }_{12}$ Ibidem, p. 27.

13 Ibidem, p. 28.

${ }_{14}$ Ibidem, p. 29.

${ }^{15}$ Foucault utiliza la expresión «régimen de verdad» para hacer referencia «a un conjunto de conocimientos reglados por la producción, la ley, la repartición, la puesta en circulación, y el funcionamiento de los enunciados». Foucault, M.: Microfísica del poder. Madrid, La Piqueta Seseña, 1980, p. 188. 
los modos de comprensión y valoración de las condiciones sociales y materiales de existencia. Mediante la activación de un dispositivo discursivo centrado en el «dejar hacer o dejar que sea el mercado», los procesos hegemónicos liberales sedimentaron su forma de concebir las relaciones de producción y gobierno.

En virtud del discurso liberal económico, el mercado debía eximirse de cualquier tipo de reglamentación o norma reguladora que delimitase su actuación ${ }^{16}$. Abogando por el axioma de "cómo no gobernar demasiado", el mercado «libre» se articuló hegemónicamente de una manera doble: por un lado, defendiendo la condición «natural» de la adecuación «precio-valor»"; por otro lado, constituyendo un espacio de verificación y falseamiento de la práctica gubernamental ${ }^{18}$. La reconfiguración de la racionalidad gubernamental que trajo consigo el liberalismo, con la consiguiente transformación de las relaciones entre el Estado y la sociedad civil, supuso una nueva forma de concebir la ciudadanía y, por ende, los ámbitos en los que se desarrollaba. A tenor de la distinción gramsciana "Estado-sociedad civil», Foucault estima que esta última se ha articulado como una tecnología gubernamental del liberalismo; si bien la sociedad civil constituye el espacio básico de intervención estatal, es decir, aparece como condición de posibilidad del Estado, paralelamente establece límites en su acción, siendo por ello potencialmente capaz de actuar como principio de oposición al Estado ${ }^{19}$. Esta paradoja, acorde con el filósofo francés, se manifiesta en la contraposición "libertad versus seguridad», situación que predispone al Estado a sufrir crisis de gobernabilidad que han desembocado en ulteriores intentos de refundación del liberalismo ${ }^{20}$.

El incipiente crecimiento de las relaciones mercantiles nacionales e internacionales, sumado a las crisis económicas y las problemáticas experiencias políticas acaecidas con el socialismo soviético, el nacionalsocialismo alemán y las políticas keynesianas durante el siglo $\mathrm{xx}$, impulsó la necesidad de reformular las nociones teóricas de la política económica. A este respecto, las escuelas de Chicago y de Friburgo se consideran dos representantes básicos de la reformulación del nuevo liberalismo, siendo la primera, con los llamados Chicago Boys, la que tuvo una mayor repercusión a nivel internacional. He ahí el campo de cultivo del posteriormente denominado «neoliberalismo» ${ }^{21}$.

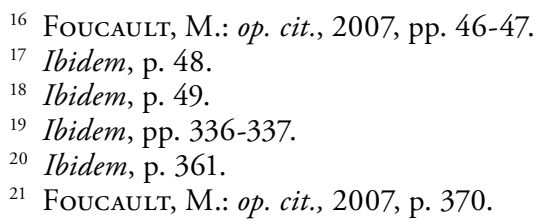




\subsection{El NEOliberalismo y la RAZÓN DE Estado}

Acorde con Foucault, el plexo teórico de los postulados del nuevo liberalismo estuvo constituido básicamente por los ordoliberales alemanes, en Europa, y los neoliberales norteamericanos, en EE. UU. ${ }^{22}$. Dada la crisis del denominado «mercado libre», los neoliberales ponderaron la necesidad de ejercer un mecanismo de regulación desde dentro y fuera del Estado en aras de garantizar el buen funcionamiento del mismo. En este sentido, las premisas teóricas cambian minuciosamente: en lugar de buscar una libertad de mercado auspiciada por el Estado, es decir, garantizada por el gobierno mediante la delimitación de su propio accionar, el ordoliberalismo propugna que sea el mercado el que, en su libre funcionamiento, se establezca como principio organizador del Estado 23.

Consecuentemente, queda invertida la tesis liberal clásica; allá donde el liberalismo se erigía como criterio delimitador del Estado, el neoliberalismo se alza como marco regulador del mismo. Así pues, en vez de cuestionar el carácter defectuoso o desigual del mercado, se ponen en evidencia los posibles efectos nocivos del Estado en materia económica. Es por ello por lo que tanto ordoliberales como neoliberales coinciden en la necesidad de actuar sobre las distintas esferas de la vida social en pos de asegurar el buen funcionamiento y competencia del mercado. Esto supone trasladar la lógica del mercado a otras esferas sociales consideradas «no económicas» tales como la familia, la natalidad, la política penal, la escuela, etc., con el propósito de disponer de un escenario formal de competencia ${ }^{24}$.

Puesto que los principios del neoliberalismo circundan en torno a cómo gobernar de acuerdo con la estructura del mercado, la racionalidad gubernamental se confecciona aceptando la necesidad de intervenir en los diversos ámbitos de la vida social. Es por ello por lo que, siguiendo la definición otorgada por Foucault, el neoliberalismo adquiere un carácter intervencionista basado en la regulación y el control. Ahora bien, cabe resaltar que la naturaleza intervencionista de sus postulados es sustancialmente diferente a formas de intervención previamente instituidas. La nueva lógica gubernamental no radica en vislumbrar qué aspectos pueden o no ser intervenidos del proceso económico; por el contrario, dejado este a la suerte de la libre competencia, las modificaciones se inscriben en aquellas dimensiones de la sociedad que inciden en su funcionamiento, como, por ejemplo, la familia, la escuela o el Estado ${ }^{25}$.

22 Ibidem, pp. 174-199.

23 «... para el neoliberalismo, el problema no era para nada saber -como en el liberalismo de tipo de Adam Smith, el liberalismo del siglo XVIII- cómo podía recortarse, disponerse dentro de una sociedad política dada, un espacio libre que sería el mercado. El problema del neoliberalismo, al contrario, pasa por saber cómo se puede ajustar el ejercicio global del poder político a los principios de una economía de mercado». Ibidem, p. 370.

24 Ibidem, p. 370.

${ }^{25}$ Ibidem. 


\section{LA ARTICULACIÓN DE LA HEGEMONÍA NEOLIBERAL A TRAVÉS DE LAS POLÍTICAS EDUCATIVAS}

Considerando que el crecimiento económico viene en paralelo al establecimiento de unas condiciones de vida adecuadas que permitan, en primera instancia, la subsistencia de los individuos, el neoliberalismo se ve en la tesitura de incorporar estrategias de acción en las distintas esferas sociales para garantizar la competencia del mercado. Dado el potencial socializador de la escuela como forjadora de ciudadanos, por un lado, y de trabajadores, por otro, esta se vuelve un espacio fundamental para la consolidación de la lógica neoliberal en la sociedad. En este sentido, las políticas educativas aparecen como instrumentos con enorme capacidad hegemónica; a través de ellas se constituyen los ciudadanos de un Estado-nación.

Teniendo en cuenta que las políticas educativas se diseñan desde el aparato gubernamental, el estudio de la escuela como Aparato Ideológico de Estado ${ }^{26}$ y, por tanto, como marco de configuración de las hegemonías neoliberales requiere un análisis del sistema político y económico en su conjunto. En este caso, el estudio discursivo de la hegemonía neoliberal implica tomar en consideración dos momentos: uno concerniente a los patrones iniciales de la articulación hegemónica que predisponen la absorción de la ideología neoliberal por parte del Estado; otro relativo a los efectos del proceso hegemónico neoliberal en el diseño de las políticas educativas. Este último aspecto supone determinar, paralelamente, hasta qué punto el espacio educativo, una vez configurado acorde con la lógica neoliberal, se erige cual fuerza hegemónica que posibilita la perpetuación del orden socioeconómico imperante.

Puesto que son tres los puntos básicos que reflejan fundamentalmente la incidencia de la hegemonía neoliberal en el ámbito escolar, a saber: la concepción del sujeto de aprendizaje, los objetivos generales del sistema educativo y la metodología de evaluación, las siguientes líneas estarán dedicadas al análisis de los mismos. Más específicamente, se estudiará de qué manera la interacción entre cada uno de estos puntos ha generado la articulación de determinados dispositivos hegemónicos.

\subsection{LA HEGEMONÍA NEOLIBERAL EN LAS POLÍTICAS EDUCATIVAS: LA LÓGICA ECONÓMICA Y LA CONCEPCIÓN DEL SUJETO}

Dado el carácter multidimensional del sistema educativo, esto es, de los distintos componentes que forman y configuran los espacios de enseñanza-aprendizaje, es menester tomar en consideración para el estudio de las hegemonías un factor protagónico: el sujeto, sea docente o discente. Si bien el sistema educacional se instituye formalmente mediante diversas sedes -escuelas, institutos y universidades-,

${ }^{26}$ Noción que extraemos de Althusser. Véase: Althusser, L.: Ideología y Aparatos Ideológicos de Estado. Buenos Aires, Nueva visión, 1974. 
la funcionalidad de las mismas depende eminentemente de los agentes implicados, de sus razonamientos y prácticas.

Bajo el paradigma neoliberal, el ser humano se concibe como un sujeto con reacciones sistemáticas, cuantificables y medibles. Siendo lo mensurable aquello que presenta cierta sistematicidad, las técnicas de estudio del comportamiento humano se fraguan en torno a la fórmula «medición-cuantificación-predicción ${ }^{27}$. Ahora bien, tomando en consideración que la aceptación e integración de una nueva concepción del sujeto requiere, según Foucault, un «régimen de verdad»o, en palabras de Gramsci, un «sentido común» que establezca cosmovisiones compartidas sobre la realidad de los individuos, cabe cuestionarse de qué manera el discurso neoliberal hizo posible la práctica sociopedagógica de acuerdo con su definición del sujeto. Remitiéndonos al análisis discursivo, observamos que la corriente psicológica del conductismo contribuyó sobremanera a la extensión de concepciones mecanicistas con respecto al comportamiento de determinados seres vivos. La confección de un marco comprehensivo del sujeto basado en la relación sistemática de ciertas variables del entorno con el comportamiento humano hizo que los postulados teóricos de Watson, primeramente, y de autores como Skinner o Hull, con posterioridad, revolucionaran la psicología en el primer cuarto del siglo $\mathrm{Xx}^{28}$. Ello tuvo como consecuencia la aparición de un modo de entender la conducta humana en términos de variables cuantificables, sujetas a estudios empíricos y susceptibles a las modificaciones del contexto.

Desde esta perspectiva, el paradigma conductista propició el forjamiento de una concepción del sujeto ajustada a la ulterior tendencia neoliberal de hacer del entorno social un espacio óptimo para los mercados; aspecto que incluía, a su vez, la modulación del comportamiento ciudadano. Teniendo en cuenta que en términos económico-neoliberales la «conducta racional» era definida por la «asignación óptima de recursos escasos a fines alternativos ${ }^{29}$, los propósitos neoliberales correlacionaron con los postulados conductistas; considerado el individuo como un ser racional que respondía sistemáticamente a los estímulos del entorno, el encauzamiento de hábitos o conductas que favorecieran las demandas del mercado se volvió pedagógicamente factible. Este carácter complementario entre la determinación contextual de la conducta y la racionalidad del sujeto económico-neoliberal contribuyó a asentar unas bases teóricas, políticas y pedagógicas, que incidieron notablemente en la manera de comprender el comportamiento humano durante el siglo xx. Puesto que eran los recursos, las tecnologías y los espacios sociales, desde la posición conductistaneoliberal, los que predisponían determinados patrones de conducta garantizadores, o no, del funcionamiento del mercado, el espacio de intervención psicopedagógica se estableció teórica y prácticamente en este nivel ${ }^{30}$.

${ }^{27}$ Ibidem, p. 307.

${ }_{28}^{28}$ Ribes, E.: El conductismo: reflexiones criticas, Fontanella, Barcelona, 1982.

${ }^{29}$ Foucault, M.: op. cit., 2007, p. 308.

30 Puiggrós, A.: Imperialismo, educación y lucha de clases, Congreso sobre Imperialismo Cultural, Argelia, 1977. 
Si nos preguntásemos, con todo, por el modo específico en el que se desplegó la hegemonía conductista-neoliberal, caeríamos en la cuenta de que ella tuvo lugar principalmente de forma enunciativa. Estableciendo una "norma de conducta», diversas pautas didácticas se incorporaron en las escuelas en aras de lograr un rendimiento escolar óptimo, adaptado a la coyuntura sociolaboral ${ }^{31}$. La lógica del paradigma conductista de aprendizaje se convirtió, en este sentido, en un instrumento teórico básico para el desarrollo de tecnologías de control escolar basadas en conceptos tales como «estímulo-respuesta», «refuerzo-castigo»o «modificación conductual».

Así pues, la alineación de enunciados teóricos conductistas en torno al marco político-económico neoliberal fraguaría un primer ejercicio hegemónico. Emergiendo el neoliberalismo como una nueva alternativa socioeconómica, sus propuestas referidas a la función social de la escuela imbricaron con las posturas del paradigma conductista. A este respecto cabe resaltar, como multiplicador hegemónico, la fuerza discursiva que supuso el auge de las denominadas «ciencias sociales», en las que tanto el paradigma conductista, desde la psicología, como el neoliberalismo, desde la economía, procuraron asentarse en pos de justificar sus supuestos. La inclusión por parte del Estado de una nueva lógica pedagógica y socioeconómica pretendió legitimarse, de esta manera, por la posición tecnócrata y científica adoptada en los discursos. A este respecto, Dreyfus y Rabinow (1982) comentan:

Las tecnologías políticas avanzan tomando lo que esencialmente es un problema político, sacándolo del ámbito del discurso político y traduciéndolo al lenguaje neutral de la ciencia. Cuando se ha llevado a cabo esto, los problemas se han convertido en técnicos, propios de especialistas [...]. Donde aparece una resistencia o un fracaso [el lenguaje de reforma] se estructura como prueba de la necesidad de reforzar y ampliar el poder de los expertos ${ }^{32}$.

\subsection{El DiSCURSO NEOLIBERAL EN LAS INSTITUCIONES EDUCATIVAS: LA EVALUACIÓN COMO TÉCNICA DE CONTROL}

Apareciendo el neoliberalismo como un nuevo modo de desarrollar políticas económicas en un contexto de crisis internacional, y dado el apogeo de unas «ciencias del comportamiento» que primaban la influencia del entorno sobre la conducta humana, nuevas formas gubernamentales toman cobertura durante el siglo xx. Remitiéndonos al carácter performativo del lenguaje, cabe especificar cuáles fueron los dispositivos enunciativos que originaron las políticas educativas sobre la base de las demandas neoliberales. Si bien el discurso conductista de la determinación del

31 Ertmet, P.A. y Newby, T.J.: «Conductismo, cognitivismo y constructivismo: una comparación de los aspectos críticos desde la perspectiva del diseño de instrucción», Performance Improvement Quarterly, 6, 1993, pp. 50-72.

32 BaLl, J.S.: «La gestión como tecnología moral: un análisis ludista», en J.S. Ball (ed.), Foucault y la educación. Disciplinas y saber. Madrid, Morata, 1997, p. 166. 
comportamiento por el contexto supuso una radical revisión de la pedagogía del sujeto, el discurso neoliberal afectó mayormente a la reestructuración de la función escolar en general.

Atendiendo a esta última idea, los discursos que suscribieron la integración de la racionalidad económica en la educación tomaron como base la necesidad de revisar la funcionalidad de la escuela. Pese a poseer una procedencia administrativoempresarial, conceptos como «eficacia», «eficiencia» o "productividad» comenzaron a aplicarse, consecuentemente, en la valoración de los sistemas educativos. Algo similar ocurrió en relación con el concepto de «evaluación», sobre el cual Martín y Reyes dicen lo siguiente:

La evaluación es un término relativamente reciente en el campo de la Pedagogía, ya que ha estado fuertemente vinculado al concepto de medida, debido a que su procedencia deriva del campo empresarial. Así, al igual que la empresa mide, cuantitativamente, los resultados alcanzados en sus procesos de producción, en el campo educativo se pretendió medir, también cuantitativamente, los aprendizajes adquiridos por el alumnado a lo largo del proceso de formación ${ }^{33}$.

Si bien a partir de los años setenta, con la aparición de paradigmas psicológicos cognitivistas y socioambientalistas, la concepción del sujeto de aprendizaje y su proceso evaluador fueron redefinidos sobre bases más cualitativas e integradoras, la estructura simbólica de la escuela continuó configurándose en torno a dispositivos discursivos de carácter mercantilista y tecnocrático ${ }^{34}$. De hecho, en la actualidad, sea la evaluación «inicial», «final», «sumativa» o «formativa», el propósito último sigue siendo, subyacentemente, el mismo: la adecuación de un perfil psicosocial específico a un entorno laboral y económico concreto. A este respecto, comenta Skilbeck:

La universidad ya no es más un lugar tranquilo para enseñar, realizar trabajo académico a un ritmo pausado y contemplar el universo como ocurría en siglos pasados. Ahora es un potente negocio, complejo, demandante y competitivo que requiere inversiones continuas y de gran escala ${ }^{35}$.

${ }^{33}$ La incorporación del concepto evaluación en educación fue promovida por primera vez por Tyler en 1942. Martín, A. y Reyes, C.I.: Fundamentos de evaluación educativa, Manual Psicopedagógico. Las Palmas de G.C., ULPGC, 2004, p. 3.

${ }^{34} \mathrm{La}$ aparición de los test psicométricos reforzó el uso de las evaluaciones cuantitativas a partir del siglo XIX. Hoskin, K.: «Foucault a examen: el criptoteórico de la educación desenmascarado", Foucault y la educación. Disciplinas y saber. Madrid, Morata, 2001, pp. 50-51.

35 SкILвеск, M.: The University challenged: A review of international trends and issues with particular relevance to Ireland. Dublin, Higher Education Authority, 2001 [versión on line parcialmente disponible en web: <http://iua.webhost.heanet.ie/publications/documents/publications/2001/ Report_11.pdf> [consulta: 19 mar. 2018]. Para ahondar en la cuestión del caso español, recomendamos también: Alonso, L.E., Fernández, C.J. y Nissen, J.M.: El debate sobre las competencias: una investigación cualitativa en torno a la educación superior y el mercado de trabajo en España. Madrid, ANECA, 2009, p. 24. 
Aceptando la concepción de la estructura social como una totalidad semiótica constituida por la producción y reproducción de significados, la institución escolar sería definible como aquel espacio de producción de conocimientos en el que las diversas etapas educativas representarían de manera simbólica los distintos niveles de una cadena de producción. La diversificación competencial por estratos curricularmente definidos y evaluativamente controlados, en este sentido, tomaría la forma del modelo administrativo-empresarial, midiendo la productividad en términos de "capital humano" ${ }^{36}$.

Teniendo en cuenta que los procesos de evaluación se dan en diferentes períodos de tiempo, destacando su función como filtro en la ascensión de los niveles académicos, el saber producido en las escuelas queda controlado sobre la base de una normatividad, medidas y criterios de actuación ${ }^{37}$. De esta manera, se genera una selección basada en una norma que permite encuadrar la interpelación de los sujetos según los objetivos pertinentes. En el ámbito educativo es predominantemente el saber el que permite la constitución de sujetos competentes; sin embargo, el conocimiento no solo interpela al sujeto como alumnado mediante la figura del profesor o el libro, sino también como especialista o experto en un área de la producción teórica o tecnológica.

Estos mecanismos de evaluación-regulación del espacio escolar, dirigidos tanto a sujetos (discentes y docentes) como a objetos (espacios y tiempos escolares, objetivos y conocimientos), si bien han sido utilizados de diversas formas siglos atrás ${ }^{38}$, representan en la actualidad el carácter operativo de la hegemonía neoliberal. En este sentido, dados los instrumentos de control académico, por un lado, y el interés neoliberal de regular el espacio gubernamental educativo, por otro, consideramos que el neoliberalismo hizo de la evaluación una estrategia para garantizar la perpetuación y ejecución de sus objetivos. Los parámetros encontrados en los informes de evaluación de la ANECA, por un lado, y las modificaciones en políticas educativas alentadas desde la esfera económico-empresarial, por otro, encarnarían tal ejercicio hegemónico ${ }^{39}$.

Uno de los ejemplos más esclarecedores a este respecto lo encontramos en el debatido «Plan Bolonia», sobre el cual Pardo señaló:

Lo que las autoridades políticas no dicen [...] es que bajo ese nombre pomposo se desarrolla en España una operación a la vez más simple y más compleja de reconversión cultural destinada a reducir drásticamente el tamaño de las universidades

36 Foucault, M.: op. cit., 2007, p. 370.

37 Ball, J.S. (comp.): Foucault y la educación. Disciplinas y saber, Morata, Madrid, 2001, pp. 8-9, 17.

${ }^{38}$ Hoskin, K.: op. cit., pp. 48-49.

39 E. Cancela estima que los tres lobbies más fuertes en lo que a las políticas europeas respecta son los siguientes: BusinessEurope, la Mesa Redonda de Industriales (ERT, por sus siglas en inglés: European Round Table of Industrialists) y la Cámara de Comercio de Estados Unidos. Véase Cancela, E.: «QQué lobbies manejan los hilos del TTIP?», La Marea, 32, 2015. doi: http://www. lamarea.com/2015/02/05/que-lobbies-manejan-en-secreto-los-hilosdel-ttip/. 
-y ello no por razones científicas, lo que acaso estuviera plenamente justificado, sino únicamente por motivos contables- $\mathrm{y}$ a someter enteramente su régimen de funcionamiento a las necesidades del mercado y a las exigencias de las empresas, futuras empleadoras de sus titulados... ${ }^{40}$.

Así pues, desde el neoliberalismo la escuela se esgrime como un escenario mercantil a nivel micro- y macrosocial, es decir: el centro educativo se establece como microcosmos de intercambio de conocimientos y cultura a través de relaciones de poder asimétricas entre profesorado y alumnado. Asimismo, se formulan las habilidades y modulan las actitudes acorde con unos parámetros preestablecidos por el currículo, el cual, confeccionado desde un espacio gubernamental específico, promueve la compra de un material didáctico transmitido y medido en términos calificadores ${ }^{41}$. Precisamente, es la calificación, en tanto que explicitación enunciativa del control evaluador, la que garantiza la consecución de los objetivos propuestos. Ahora bien, en la medida en que estos objetivos son formulados para atender al mercado, la escuela aparece como una pieza estratégica clave del macrocosmos social.

En coherencia con esta última idea puede observarse cómo, dentro de la estructura social general, la escuela juega un rol esencial en la integración y expansión de la lógica mercantil; lógica que desde el discurso político incluye enunciados como «recorte del gasto público» o «riqueza social» ligados a conceptos de índole empresarial como «eficacia», «evaluación» o "productividad». Esta lógica de las equivalencias neoliberal, acorde con nuestros análisis, sería fruto de un proceso de articulación hegemónico en el que el enunciado "funcionamiento óptimo" constituiría una cadena de equivalencias con las nociones de "eficacia», "eficiencia» y «productividad». Los «costes» y la «evaluación» serían, por su parte, los criterios de regulación de dicho «funcionamiento óptimo».

Este hecho se hace notar en publicaciones tales como las del Boletín Oficial de la Junta de Andalucía, cuando establece:

Los programas universitarios deben estructurarse de manera que se mejore directamente la empleabilidad de los licenciados. Las universidades deben ofrecer planes de estudio, métodos docentes y programas de formación o readiestramiento innovadores que, a las capacidades más propias de la disciplina, sumen otras de carácter más amplio relacionadas con el empleo [...]. Incluso se pretende ir más allá de las necesidades del mercado de trabajo y estimular una mentalidad emprendedora entre los alumno ${ }^{42}$.

Por otro lado, en la medida en que el desarrollo estructural y la disponibilidad de los recursos sociales dependen de fuentes de financiación, las decisiones políticas -incluidas las educativas- quedan delimitadas por las demandas y

\footnotetext{
40 Véase Pardo, J.L.: «La descomposición de la Universidad», El País, 2008.

${ }^{41}$ Hoskin, K.: op. cit., pp. 33-56.

42 Boletin Oficial de la Junta de Andalucia, 146, 2007.
} 
exigencias de los mercados, más comúnmente reconocidos mediante el nombre genérico «El Mercado»" . Esta noción de «El Mercado» instituida como un agente abstracto resulta fundamental de cara a la lucha hegemónica, pues, si bien posee el potencial de interpelar a los sujetos como "ciudadanos racionales», "consumidores» $\mathrm{y}$ «trabajadores», no permite ser reconocida en la especificidad de sus actores. En este sentido, la multiplicidad de las acepciones que engloba «El Mercado» disemina toda significación de responsabilidades y, con ello, dificulta la construcción de un proceso contrahegemónico preciso.

\section{3. «El MERCADO» COMO MECANISMO REgULAdOR DE LOS ObJETIVOS EDUCATIVOS: DESDE LA DEFINICIÓN DEL SUJETO DE APRENDIZAJE HASTA LA EVALUACIÓN DEL SISTEMA ESCOLAR}

Si atendemos al carácter pluridimensional de los objetivos educativos constataremos que cuanto más alta es la etapa académica, más concretos son los programas diseñados para responder a las competencias exigidas por «El Mercado» ${ }^{44}$. Puesto que «El Mercado» requiere unos sujetos de aprendizaje valorados sobre parámetros de «utilidad social», esto es, sujetos que contribuyan al aumento del PIB ${ }^{45}$, este se convierte en un criterio de ordenamiento sociopolítico. Así pues, la tesis de Foucault sobre la concepción del mercado como mecanismo de regulación gubernamental sería trasladable al ámbito político-educativo; la pretensión de modular los espacios académicos en aras de hacer posible la existencia competitiva de un «mercado libre» hace de este un criterio central de regulación pedagógica o escolar.

La nueva concepción del sujeto de aprendizaje y la necesidad de reformular los objetivos del sistema educativo en términos administrativo-empresariales se presentan, desde el discurso político, con una nomenclatura aparentemente «neutral», remitiendo a la ciencia como soporte teórico de sus proposiciones ${ }^{46}$. El discurso neoliberal se convierte, de este modo, en legitimador de unas prácticas educativas específicas desde el marco de la racionalización científica o tecnocrática ${ }^{47}$. Consecuentemente el neoliberalismo, en virtud de su reformulación del espacio de intervención estatal, produce relatos que cimientan en la consciencia de la sociedad civil

43 Para profundizar en las consecuencias sociales que tiene el libre mercado, véase GALBRAIth, J.K.: El dinero: de dónde vinoladónde fue. Barcelona, Ediciones Orbis, 1983.

${ }^{44}$ Las modificaciones de política educativa más acentuadas de los últimos años se han realizado principalmente a nivel de ESO y sistema universitario; la LOGSE, la LOE y la LOMCE, para el primer caso, y el Plan de Bolonia, para el segundo caso, son algunos ejemplos de ello.

${ }_{45}$ El propio J.E. Stiglitz (premio Nobel de economía) afirmó en 2008 que el PIB compensa particularmente a los gobiernos y su producción material, descuidando aspectos relacionados con el medio ambiente o el bienestar ciudadano. J.E. Stiglitz, A. Sen y J.P. Fitoussi, «Informe de la comisión sobre la medición del desarrollo económico y del progreso social», París, 2008, doi: http://www.palermo.edu/Archivos_content/2015/derecho/pobreza_multidimensional/bibliografia/Biblio_adic5.pdf.

46 BALL, J.S.: op. cit., p. 166.

47 Ibidem., p. 28. 
una lógica mercantil. Apoyándose en la expresión «crisis del Estado-nación» como elemento antagónico del cual alejarse, el discurso neoliberal proyecta un «deber ser» revestido de cientificidad dirigido a modular la prâxis política.

Asimismo, allá donde los economistas neoliberales de Friburgo y Chicago incidieron en la racionalidad gubernamental, el paradigma conductista incidió en la racionalidad pedagógica entroncando con la noción del sujeto racional neoliberal. Cabe subrayar que el discurso conductista, en tanto que paradigma psicológico, adquiere un potencial hegemónico determinante en la medida en que la noción «sujeto de aprendizaje» es extrapolable a otras subjetividades no puramente académicas, pues todo sujeto es aprendiz a lo largo de su experiencia cognitiva y socioafectiva. A este respecto, el neoliberalismo se articularía en torno al siguiente razonamiento: «Si todo sujeto es un sujeto de aprendizaje, este es capaz de aprender a calcular razonadamente $y$, por ende, a funcionar social y mercantilmente de manera adecuada». Ahora bien, esta nueva forma de entender al sujeto, una vez integrada en la política educativa, tiene como condición de posibilidad la evaluación, es decir, el control.

En coherencia con lo anterior, el mecanismo de regulación educativa por antonomasia viene a ser representado por los procesos formales de evaluación, pues a través de esta se revela, en última instancia, la condición de ser del sujeto. La evaluación se establece, así, como un instrumento descriptivo que valora "lo que es» (proceso de enseñanza-aprendizaje) sobre una normativa prescriptiva (currículum) de «lo que debería ser» (correspondencia formación-mercado). Un aspecto a destacar de este mecanismo de control es su posibilidad de autogestionarse; los propios agentes evaluadores, principalmente inspectores y docentes, llegan a aplicar la evaluación sobre su propia actividad en pos de vigilar funcionalmente su adecuación en la cadena de la producción escolar.

\subsection{LOS NIVELES DE ARTICULACIÓN DEL PROCESO HEGEMÓNICO NEOLIBERAL}

Los análisis anteriores nos permiten constatar, en suma, dos niveles paralelos e interdependientes a través de los cuales la hegemonía neoliberal se desarrolla: un nivel de contenido relativo a la carga simbólica de los discursos, esto es, la concepción del sujeto racional, la normativización de unos objetivos a seguir y la concreción de mecanismos reguladores que velen por la operatividad del proceso; un nivel procedimental, relacionado con las acciones y los espacios a través de los cuales los discursos operan. Este nivel procedimental se constituye, a su vez, por dos momentos: uno referido al surgimiento del proyecto neoliberal como propuesta de mejora social justificada por la defensa del «mercado libre»; otro concerniente a la aceptación de la lógica neoliberal como una nueva forma de gobierno y su reflejo en la normativa política. Veamos en la página siguiente un cuadro-resumen de todo ello: 
OBJETIVO NEOLIBERAL = PUNTO NODAL DE REPRESENTACIÓN GENERAL

Procurar el buen funcionamiento/competencia de El Mercado a través de la intervención en las distintas esferas de la vida social; en este caso, contextos escolares/académicos.

CONDICIONES SOCIALES DE PRODUCCIÓN DEL DISCURSO

\begin{tabular}{|c|c|c|}
\hline $\begin{array}{c}\text { ARTICULACIÓN DE DEMANDAS PARTICULARES } \\
\text { Necesidad de renovar las políticas económicas } \\
\text { tras un período de crisis. } \\
+ \\
\text { Necesidad de mejorar el espacio educativo } \\
\text { para la formación de sujetos con capacidad } \\
\text { de acceso al mercado laboral. } \\
+ \\
\text { Ímpetu de hacer del estudio del } \\
\text { comportamiento humano una ciencia. } \\
\text { LóGICA DE LAS EQUIVALENCIAS }\end{array}$ & $\begin{array}{l}\longrightarrow \\
\longrightarrow \\
\longrightarrow\end{array}$ & $\begin{array}{l}\text { Principales SUjeTOS DE ENUNCiación } \\
\text { Escuela de Friburgo (Hayek). } \\
\text { Escuela de Chicago (Friedman). } \\
\text { Política educativa demarcada } \\
\text { por el Plan de Bolonia. } \\
\text { Psicología conductista: } \\
\text { Watson, Skinner. }\end{array}$ \\
\hline \multicolumn{3}{|c|}{ ELEMENTOS SEMIÓTICO-IDEOLÓGICOS DEL DISCURSO } \\
\hline $\begin{array}{r}\text { Noción General QUE TRASCIEN } \\
\text { El sistema educativo debe ser regulado } \\
\text { de acuerdo con la }\end{array}$ & $\begin{array}{l}\text { E E INTH } \\
\text { en pos d } \\
\text { deman }\end{array}$ & $\begin{array}{l}\text { LAS DEMANDAS PARTICULARES } \\
\text { ear sujetos racionales y competentes } \\
\text { le El Mercado. }\end{array}$ \\
\hline
\end{tabular}

Concepción DEL SUJETo RACiONAL (NEOliberalismo)

Sujeto que realiza una asignación óptima de recursos escasos a fines alternativos; calcula los diversos actores sociales y las consecuencias de sus actos.

$$
+
$$

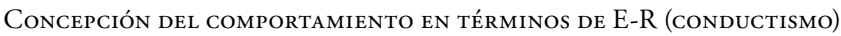

La conducta es el resultado de la interacción del sujeto-entorno; controlando el contexto se determina la conducta.

Posibilidad de instruir, medir, evaluar y controlar la conducta en aras de transformar la sociedad.

Cadena de equivalencias: eficacia-evaluación-productividad-racionalidad-riqueza social. LÓGICA DE LAS DIFERENCIAS: ineficacia-descontrol-irracionalidad-crisis-gasto público.

\section{LA HEGEMONÍA NEOLIBERAL EN LA ESCUELA: ÁREAS DE INTERVENCIÓN}

- A nivel del sujeto (discente): concepción del sujeto de aprendizaje; potencialmente maleable (enseñable = dócil); división socio-técnica del trabajo para garantizar la «eficacia».

[De la teoría a la práctica]

- A nivel del sujeto (docente): equiparación de la productividad con el salario.

[De la teoría a la práctica]

- A nivel del objeto (saberes, instrumentos y estructuras): formas de evaluación que garantizan la eficacia explicitada en objetivos.

[Desde la teoría sobre la teoría y la práctica]. 
Tomando nuestro esquema como referencia, las estrategias discursivas y tecnologías de control a través de las cuales «El Mercado» actúa como criterio de regulación de las políticas educativas son, en conclusión, las siguientes:

a) Estrategias discursivas

- Lógica de la diferencia: se utiliza como oposición discursiva la "crisis», el «caos», la «irracionalidad», la «mala gestión gubernamental» o la «ineficacia del sistema educativo anterior».

- Lógica de la equivalencia: se establecen objetivos político-educativos mediante discursos que incluyen conceptos y expresiones tales como «racionalidad», «orden», «administración adecuada de gastos públicos», «educación centrada en las demandas de "El Mercado"".

b) Tecnologías de control

- Currículum como tecnología de control y concreción de las políticas educativas: explicitación normativa de los objetivos, así como de los contenidos y metodologías a desarrollar en todo espacio académico formal ${ }^{48}$.

- Evaluación como tecnología de control:

- La cadena de equivalencia «eficacia-competencia-productividad-rendimiento escolar óptimo" se establece como criterio de evaluación.

- La evaluación, como forma de garantizar el cumplimiento de los objetivos, se dirige tanto a sujetos como a objetos o a ella misma:

- Evaluación centrada en los objetos (saberes, objetivos y estructuras): este tipo de evaluación muestra si existe necesidad de modificar los elementos estructurales del sistema educativo. La atención no recae en los sujetos sino en los materiales o propuestas que utilizan.

- Evaluación centrada en los sujetos (discentes): controla la correspondencia entre los objetivos a alcanzar y los logros por etapa bajo un mismo principio «normalizador».

- Evaluación centrada en los sujetos (docentes): evaluación sobre las calificaciones emitidas, la formación del profesorado y los métodos didácticos. El inspector es el agente evaluador principal.

- Autoevaluación y metaevaluación: si bien la primera implica la evaluación del sujeto sobre sí mismo, la segunda supone evaluar el propio procedimiento evaluador ${ }^{49}$.

48 Denominamos «educación formal» a aquel proceso educativo desarrollado dentro del marco legislativo estatal; todo proceso de enseñanza-aprendizaje que esté fuera del tal marco se definiría como educación informal (p. ej., actividades extraescolares) o no-formal (p. ej., educación para adultos), según el caso. Fernández, C.M. y Rodríguez, M.C.: «Educación formal, no formal e informal en el espacio europeo: nuevas exigencias para los procesos de formación en educación", Aula Abierta, 2005, pp. 45-56.

49 Allá donde la autoevaluación supone que el sujeto/objeto evaluador y evaluado es el mismo, la metaevaluación implica evaluar el propio procedimiento de evaluación. MARTín, A. y Reyes, C.I.: op. cit., pp. 10-16. 


\section{POSIBILIDADES CONTRAHEGEMÓNICAS}

Teniendo en cuenta los análisis anteriores, podemos decir que el ejercicio hegemónico neoliberal se desarrolla a partir de tres elementos claves, a saber: el sujeto, la sociedad político-civil y el mercado. De ahí que la fuerza de su acción haya residido principalmente en la articulación de dispositivos discursivos operantes en estos tres frentes; por un lado, generando consensos sobre la racionalidad y el aprendizaje del sujeto de la mano de paradigmas psicológicos tales como el conductismo; por otro lado, estableciendo una propuesta de cambio social que, diferenciándose de un contexto de crisis, toma «El Mercado» como criterio regulador de la adecuada organización gubernamental.

Ahora bien, aceptando la preponderancia actual de la lógica neoliberal en la racionalidad gubernamental espańola y, por ende, en el desarrollo de las políticas educativas, cabe cuestionarse sobre dos aspectos interdependientes: las características del sujeto neoliberal y la posibilidad de generar una estrategia contrahegemónica ${ }^{50}$ a partir de la esfera escolar. Si bien atender la primera cuestión supone discernir los mecanismos psicológicos que imperan en el accionar de los individuos, motivándolos a perpetuar o, en su caso, a contradecir las condiciones sociales de existencia, el segundo punto requiere desentrañar el margen de acción emancipadora que desde los espacios académicos puede fraguarse. Vayamos por pasos.

Acorde con las consideraciones de Gramsci y Althusser, la «lógica neoliberal», en tanto que práctica hegemónica y discursiva, constituye subjetividades políticas y formas de concebir la actuación del Estado a través de la interpelación ideológica. Instituido «El Mercado» como marco regulador de la prâxis gubernamental y criterio demarcador del «deber ser» social, los ciudadanos, en aras de posibilitar el funcionamiento de la sociedad, se ven instruidos a integrar unos patrones de pensamiento y conducta consonantes con la doctrina económica neoliberal. En coherencia con ello, la racionalidad neoliberal puede definirse como una lógica político-económica o tecnología de gobierno ${ }^{51}$ que, más allá de ocultar realidades, articula «verdades» $\mathrm{y}$ «razones de ser» que determinan el ordenamiento del Estado, así como de instituciones tales como la escuela.

Lo que en este punto hemos de tener en cuenta es que dichas «razones de ser» o pautas de racionalidad, aunque operen desde la funcionalidad del sistema, sea escolar, sea gubernamental, recaen enteramente sobre los sujetos. En efecto: la función del sistema depende de la operatividad de sus partes componentes; en este caso, los sujetos. En virtud de ello, diríamos que la lógica de equivalencia que se establece entre el ámbito empresarial y los espacios educativos tiene como soporte principal la sinergia de las subjetividades implicadas. En la medida en que la escuela

\footnotetext{
50 Se entiende por «contrahegemonía» aquel proceso antagónico a la articulación hegemónica dominante. SADER, E.: «Hegemonía y contra-hegemonía para otro mundo posible», Rebelión, 2001.

${ }^{51}$ Foucault, M. op. cit., 2007, pp. 336-337.
} 
constituye, justamente, los procesos de configuración de los distintos perfiles sociales y profesionales, ella se vuelve uno de los escenarios emancipadores más potentes ${ }^{52}$.

He ahí la razón por la que, de existir algún tipo de oportunidad contrahegemónica, ella deba encontrarse en los propios sujetos escolares; más concretamente, en sus modos de operar de manera racional y afectiva. Aclarado esto, cabe retomar nuestra pregunta inicial: ¡cuál es el perfil psicopedagógico y profesional que se potencia actualmente en el sistema educativo, predominado por la racionalidad neoliberal? La respuesta no se hará esperar: la lógica del mercantilismo aplicada a la generalidad de la sociedad desemboca en lo que se ha venido a denominar homo economicus $^{33}$. Teniendo en cuenta que, según Foucault, el homo economicus es un producto social de la tecnología gubernamental liberal, este se define por ser un sujeto de interés, esto es, un sujeto guiado por intereses eminentemente lucrativos e individuales ${ }^{54}$. En este sentido, dada la definición que otorga el neoliberalismo del comportamiento racional, homo economicus y «sujeto de interés» vendrían a constituir dos expresiones particulares de la subjetividad neoliberal.

Teniendo en cuenta la peculiaridad de la misma, la estrategia contrahegemónica que desde la escuela convendría confeccionar implicaría una educación enfocada en valores como el altruismo, la filantropía o el compañerismo. Ello supone examinar, paralelamente, de qué manera resultan compatibles la racionalidad del homo economicus y una ética que vele por la libertad tanto como por la igualdad social. En otras palabras: el proceso contrahegemónico del que hablamos pasa por analizar qué tipo de ética o de comportamiento moral ${ }^{55}$ tiene cabida en la racionalidad del sujeto neoliberal. Y es que, si el sujeto neoliberal fuera éticamente compatible con el sujeto de derecho, el propósito de la estrategia contrahegemónica no estribaría tanto en la erradicación del homo economicus cuanto en su modificación.

En aras de responder a esta cuestión, nos apoyaremos en los análisis foucaultianos sobre el homo economicus y el «sujeto de derecho» (civil y moral). Lo que ellos explicitan queda puesto en evidencia de forma clara: el «sujeto de derecho», en contraste con el homo economicus, es un sujeto centrado en el deber social de los gobernantes y en la garantía de unas condiciones sociales basadas en la seguridad y la justicia; en última instancia, él tiende a primar la igualdad colectiva antes que la libertad individual ${ }^{56}$. De ahí que el «sujeto de derecho» se constituya como el contrario dialéctico del homo economicus. Más específicamente, diríamos que la principal diferencia entre estas dos categorías de la subjetividad radica en la carac-

52 Gramsci, A.: Cuadernos de cárcel: tomo 2. Edición crítica del Instituto de Gramsci, Valentino Gerratana (ed.), Roma, 1999, pp. 55, 195.

53 «El 'homo economicus' es, si se quiere, el punto abstracto, ideal y puramente económico que puebla la realidad densa, plena y compleja de la sociedad civil. O bien: la sociedad civil es el conjunto dentro del cual es preciso resituar esos puntos ideales que constituyen los hombres económicos, para poder administrarlos de manera conveniente». Foucault, M.: op. cit., 2007, p. 337.

54 Ibidem, p. 316.

55 García, P.: «Ética y moral», Diccionario Filosófico, 1999, doi: http://filosofia.org/filomat.

56 Foucault, M.: op. cit., 2007, p. 317. 
terización del "principio de transferencia» de los derechos o, en otras palabras, en la capacidad para fundamentar las limitaciones de la propia acción en el respeto de los derechos ajenos ${ }^{57}$.

A este respecto, cabe subrayar que «no solo hay una heterogeneidad formal entre el sujeto económico y el sujeto de derecho», sino que también hay una diferenciación en sus modos de relacionarse con el poder político ${ }^{58}$. Así pues, mientras que el «sujeto de derecho» fundamenta su existencia en una racionalidad gubernamental jurídica y, por ende, demanda la supervisión de las desigualdades sociales, el homo economicus aboga por una desregulación del juego económico y, paradójicamente, una regulación en lo sociofamiliar, cultural y político-educativo. Por tanto, de responder la racionalidad del sujeto neoliberal a alguna moral, esta tendría un carácter eminentemente instrumentalista o utilitarista, pues la disposición de los sujetos estaría principalmente motivada por la optimización de los propios intereses, primando los fines, ganancias o beneficios, sobre los medios utilizados para lograrlos.

En coherencia con ello, diríamos que el principal reto del proyecto contrahegemónico estriba en la inculcación de una ética basada en el altruismo, en vez del lucro, en el compañerismo, en vez de la competencia, y en la vocación, en vez de la instrumentalización de los perfiles profesionales. El ideal sería, en cualquier caso, alcanzar un equilibrio entre la libertad individual, incluyendo la posibilidad de generar riqueza personal, y la igualdad social, que se traduce en la aplicación de estrategias que compensen las brechas culturales existentes entre los distintos sectores de la población.

Ahora bien, la promoción de los valores éticos mencionados debe combinarse, si se quiere fraguar una estrategia emancipadora adecuada, con un contenido educativo determinado. A este respecto, encontramos dos grandes tradiciones pedagógicas: la fraguada por Platón, que sintonizaría con los ideales de la socialdemocracia, y la confeccionada por los sofistas, que convergería con la lógica neoliberal. En efecto: allá donde la primera favorece un contenido pedagógico basado en la transversalidad y universalidad del conocimiento teórico $0^{59}$, la segunda promueve la particularidad y utilidad de la aplicación o la técnica ${ }^{60}$. En este sentido, el mencionado proyecto contrahegemónico debería priorizar el amor por el conocimiento (philosphía) y no la mera utilidad del mismo. No se trata, empero, de negar una cosa por otra, o de eliminarla, sino de reorganizar la jerarquía de los valores pedagógicos.

Téngase en cuenta, con todo, que las sucesivas reformas educativas hasta ahora desarrolladas manifiestan la pugna existente entre una comprensión socialdemócrata de la educación y la posición neoliberal. Si la primera ha posibilitado la

57 Ibidem, p. 316.

58 Ibidem, p. 318.

59 De este modo, la contraposición dôxa-epistéme de Platón puede considerarse correlativa a la contraposición ideología-ciencia de Althusser.

${ }^{60}$ Lozada, B.: Foucault: poder, discurso y sofistica. Bolivia, UMSA, 2000, pp. 247-251, doi: http://www.cienciasyletras.edu.bo/publicaciones/filosofia/articulos/estudios\%20bolivianos\%20II/ Estudios\%20Bolivianos\%202.pdf. 
movilidad -generalmente ascendente-de los diversos grupos sociales en la estructura político-económica, la segunda ha desencadenado la creación de contextos educativos mayormente selectivos, de carácter tecnócrata y mecanicista. El logro de un acuerdo legislativo entre los distintos partidos políticos se vuelve, en coherencia con lo anterior, más que necesario.

\section{CONCLUSIÓN}

La pugna para definir la política educativa constituye [...] una lucha por el control de las funciones de producción y reproducción de la enseñanza ${ }^{61}$.

Partiendo de los análisis desarrollados a lo largo de las páginas anteriores, diversas son las reflexiones y conclusiones a extraer.

En primer lugar, teniendo en cuenta los inicios ilustrados de la escuela contemporánea, podemos decir que el aparato escolar ha estado desde el siglo XVIII intrínsecamente unido al Estado, de tal forma que su actividad ha quedado siempre sujeta a las políticas educativas gubernamentalmente confeccionadas. Asimismo, de acuerdo con las aportaciones teóricas del filósofo francés Althusser, así como del italiano Gramsci y el argentino Laclau, el estudio de la función social del sistema educativo nos ha permitido constatar el rol de este como Aparato Ideológico de Estado y, por ende, como espacio de articulación hegemónica y construcción discursiva de subjetividades.

Más específicamente, el Análisis del Discurso y la concepción del sistema social y sus diferentes ámbitos como escenarios simbólico-discursivos han contribuido significativamente a la comprensión del ejercicio hegemónico como integrador de demandas sociales plurales en torno a un proyecto político particular. En este caso, desde el tercer cuarto del siglo xx las estrategias de hegemonía neoliberal se han constituido principalmente mediante un tipo de discurso lingüístico, siendo los enunciados cientificistas de la economía, por un lado, y de la psicología conductual, por otro, los instauradores de una nueva racionalidad del sujeto social y pedagógico respectivamente.

La exploración discursiva, lingüístico-semiótica, de los procesos hegemónicos neoliberales en el ámbito educativo nos ha posibilitado advertir dos tipos de influencia: una primera relativa a la concepción y evaluación de los sujetos, discentes y docentes; una segunda referida a la función social y la finalidad del sistema educativo o los entornos académicos. Precisamente, la lógica del funcionamiento empresarial constituiría la relación que ambas esferas guardan entre ellas. Y es que, en efecto, sujeto (docente o discente) y objeto (institución o técnica) se fundamentan mutuamente sobre la base de dispositivos de control neoliberal.

${ }^{61}$ Kinght, J., Smith, R. y SAchs, J.: «La deconstrucción hegemónica: política multicultural y respuesta populista», en BALL, J.S. (ed.): Foucault y educación. Disciplinas y saber. Madrid, Morata, 2001, p. 145. 
En concordancia con lo anterior, hemos podido comprobar el uso, por un lado, del término "eficacia» como criterio de evaluación interna del espacio académico particular y, por otro lado, del principio de "adaptación» al mercado como mecanismo de regulación externa del sistema educativo general. Las políticas educativas serían, en este orden de ideas, manifestaciones materiales de "El Mercado" como norma constituyente de una nueva racionalidad gubernamental. De ahí que, de ser trasladada legislativamente a la escuela la lógica socioeconómica propia del neoliberalismo, sean las políticas educativas y, más específicamente, el currículum las vías de acceso formalmente determinadas; vías aquí concebidas como estructuras simbólicas que reflejan los efectos del proceso hegemónico neoliberal.

Por su parte, dada la primacía de la hegemonía neoliberal en la racionalidad política general y en el marco pedagógico en particular, se ha analizado qué tipo de subjetividades tienden a fraguarse en el espacio académico. Teniendo en cuenta que la lógica neoliberal ha incidido en la concepción de los sujetos de aprendizaje, en el uso de los mecanismos de evaluación y en el diseño de objetivos académicos, ella ha contribuido a la incubación del denominado homo economicus; se trata de un tipo de subjetividad que prima el lucro individual frente a la igualdad social. De ahí la importancia que la reeducación del homo economicus, en cuanto agente de la perpetuación del orden neoliberal, tiene en toda estrategia contrahegemónica.

En efecto: en el momento de barajar estrategias para la construcción de un proyecto contrahegemónico, más que abogar por un cambio en las políticas o instituciones educativas - lo que supondría actuar en la dimensión estructural-, se considera oportuno optar por una transformación progresiva que actúe sobre el plano ideológico, esto es, sobre la funcionalidad de las consciencias, las cosmovisiones y los sentidos comunes (en última instancia, sobre el sujeto)

Llegados a este punto, cabe preguntarse ¿en qué medida es posible transformar la racionalidad gubernamental desde la educación formal, es decir, desde un ámbito regulado por el propio gobierno? Se trata de una pregunta cuya respuesta se fundamenta en otra que ha subyacido en todos nuestros análisis: ¿̨hasta qué punto el Estado, en cuanto ámbito dominado por la lógica neoliberal, pierde la legitimidad de sus acciones y decisiones? Y es que, si los mecanismos de regulación neoliberal determinan la dirección gubernamentel del Estado, el ejercicio soberano de este, más allá de residir en los representantes legítimamente electos, queda sujeto a presiones de lobbies e intereses comerciales que, en última instancia, reflejan un juego de relaciones de poder geopolíticas y económicas ajeno a las urnas ${ }^{62}$.

Precisamente, es este cuestionamiento de la legitimidad soberana el que fundamenta el proceso contrahegemónico que desde la escuela pudiera fraguarse. Con todo, en la medida en que la posibilidad de la contrahegemonía neoliberal

62 Si bien el citado Plan de Bolonia ejemplifica el poder de «El Mercado» en el caso educativo, la reforma constitucional (art. 135) en torno a los presupuestos estatales fraguada el verano de 2011 por el presidente del gobierno del momento (J.L. Zapatero) y el líder de la oposición (M. Rajoy) refleja el poder de la presión tecnócrata-bancaria. 
parte del cuestionamiento de la legitimidad gubernamental, la necesidad de forjar un diálogo crítico y reflexivo desde diversos frentes -filosófico, sociológico, jurídico y pedagógico- se torna ya no solo oportuna, sino imperiosa; la calidad de nuestra democracia depende de ello.

Recibido: mayo de 2018, Aceptado: junio de 2018 
